\title{
The Effects of Time to Fall Asleep and Sleep Duration on Short-term Memory Performance in Chinese High School and College Students
}

\author{
Xinzhu Xiang ${ }^{1 *}$ \\ ${ }^{1}$ College of the Environment, Forestry \& Natural Sciences, Northern Arizona University, Flagstaff, Arizona 86011, \\ China,xx38@nau.edu
}

\begin{abstract}
Previous researches have noticed the Chinese high school and college students putting off bedtime and shortening the sleep duration under an intense education system. However, several studies have reported the importance of a moderate sleep pattern. The exam-oriented education system prevalent in China is largely lecture-based which depends a lot on memorization. Evidence suggests that an adequate sleep pattern can optimize memory performance. The aim of the present study was to investigate the effects of time to fall asleep and sleep duration on short-term memory performance for 10 consecutive days in Chinese high school and college students $(\mathrm{N}=61$, mean age $\pm \mathrm{SD}=18.85 \pm 2.03$ years; 25 males). No significant correlation between the time to fall asleep and memory test performance was found $(\mathrm{P}=0.1062)$. When sleep duration was between 6.5 to 7.5 hours, sleep duration had a significantly positive correlation with memory test performance $(\mathrm{P}<0.001$, rho $=0.41)$. When sleep duration was between 7.5 to 8.5 hours, sleep duration had a significant negative correlation with memory test performance $(\mathrm{P}<0.001$, rho $=-0.47)$. These findings suggest 6.5 to 7.5 hours of sleep is sufficient for optimal short-term memory performance. Further experimental studies are needed to support the current study's findings.
\end{abstract}

Keywords: Chinese, high school students, college students, time to fall asleep, sleep duration, short-term memory.

\section{INTRODUCTION}

Chinese students have been experiencing prolonged and intense academic pressure since their early school years under an exam-oriented education system. Consequently, putting off bedtime and shortening sleep duration in order to meet academic demands become prevalent among Chinese high school and college students $^{[1,2,3]}$. However, researchers have also verified that inadequate sleep is associated with difficulty in concentration and poor work performance ${ }^{[4,5]}$.

Exam-oriented education has been hitherto prevalent in China. This conventional mode of schooling is largely lecture-based, therefore, it focuses a lot on memorization. There are three categories of memory: sensory memory, short-term memory, and long-term memory ${ }^{[6]}$. This study in particular focuses on short-term memory which is constantly employed in a school setting: remembering what teachers wrote down on the board, taking notes on teachers' dictated contents, and writing down the poetry students looked through a few minutes before the Chinese exam. Previous literature also suggests that short-term memory is the basis of higher-order operations such as troubleshooting, problem-solving, and developing plans $^{[7]}$. If we can better understand the key factors that impact short-term memory, it could have valuable implications for Chinese students and educators in the context of the examination-orientated education system.

Since adequate sleep is an important factor to optimize memory processes ${ }^{[8,9,10]}$, the first aim of this research is to investigate the correlation between sleep patterns (time to fall asleep and sleep duration) and short-term memory performance. It is hypothesized that 1) putting off the time to fall asleep will significantly compromise the short-term memory performance; 2) Longer sleep duration will significantly improve the short-term memory performance. If it is determined that time to fall asleep (TFA) and sleep duration (SD) are linked with short-term memory test performance (MT), it could provide essential implications to Chinese educators and students. 


\section{METHOD}

\subsection{Participants}

A total of 62 Chinese students were invited to participate as convenience samplings in this study. Participants consisted of 32 high school students and 30 college students. One college student quitted the study, thus, the final sample included 61 participants (mean age $\pm \mathrm{SD}=18.85 \pm 2.03$ years; 25 males $)$

\subsection{Ethics Statement}

Written informed consent was obtained from all participants. All the parents/caregivers of the participants under 18 years of age signed consent forms for their child to participate in the current study.

\subsection{Design and Materials}

Testings were conducted in a self-reported mode for 10 consecutive days. Participants were instructed to download the Snail Sleep app (Apple or Android supported). On the days of the experiment, participants pressed "Start" on the Snail Sleep app and put the phones next to their pillows after they went to bed. The Snail Sleep app kept monitoring their sleep until the participants pressed "Stop" on the Snail Sleep app when they woke up the next morning. One hour after the participants woke up, they accessed the short-term memory test (Link to the test: https://humanbenchmark.com/tests/number-memory). In the short-term memory test, digits were presented at a rate based on the length of the digits. At the end of the digits sequence, an input field was displayed, and participants typed in the order in which the digits had appeared. Below the input field was a "Submit" key that participants pressed after a recall had been completed. The length of the digits increased by one with the test level increasing by one. Testing continued until the participants failed to recall the digits at that length completely correct. The level that the participant ended at was the score of the memory test. Each participant was required to do the memory test twice in a row each experimental day. The numeric average of two scores represented his/her final short-term memory test score on that day. The participants generally completed the online numeric working memory test in 5 to 10 minutes. The same procedure was repeated for 10 consecutive days.

\section{STATISTICAL ANALYSIS}

To test the normality of TFA SD, and MT, the Shapiro test was performed and the alpha level was set at 0.05 . If the p-value was less than 0.05 , the null hypothesis was rejected and there is evidence that the data tested are not normally distributed. Then spearman test would be applied to test the association between TFA and MT, and between SD and MT. If the p-value was larger than 0.05, the null hypothesis was not rejected and there is evidence that the data are normally distributed. Then the Pearson $r$ test would be applied to test the association between TFA and MT, and between SD and MT. Significance was set at $\mathrm{P}<0.05$ for both the Spearman test and Pearson $r$ test. The absolute value of the Pearson product-moment correlation coefficient ( $r$ ) and the absolute value of the Spearman rank correlation coefficient (rho) was used to describe the strength of the correlation. The correlation was very weak (rho or $r=0.00-0.19$ ), weak (rho or $r=$ $0.20-0.39$ ), moderate (rho or $r=0.40-0.59$ ), strong (rho or $r=0.60-0.69$ ), and very strong (rho or $r=0.80-1.0)^{[11]}$.

\section{RESULTS}

61 participants self-reported SD, TFA, and MT data for 10 consecutive days. Because of forgetting to turn on the sleep monitor app or to do the memory test $(n=19)$, the final data pool obtained 591 data sets of SD, TFA, and MT.

A Spearman correlation coefficient revealed no significant correlation between $\mathrm{TF}$ and $\mathrm{MT}(P=0.1062$, rho $=-0.067)$. Analysis of the spearman correlation coefficient indicated that no significant correlation was found between SD and MT $(P=0.8843$, rho $=-0.006)$.

\subsection{ADDITIONAL ANALYSIS}

Some participants self-reported that both too long and too short sleep duration compromised their short-term memory test performance. However, there is not a universally standard criterion for optimal sleep duration $^{[12,13]}$. The National Sleep Foundation recommended teenagers (14-17 years of age) sleep 8 to 10 hours every day, and 7 or 11 hours may be an appropriate sleep duration ${ }^{[12]}$. Young adults (18-25 years of age) were recommended to sleep 7 to 9 hours, while 6 or 10-11 hours may be appropriate ${ }^{[12]}$. The sufficient sleep duration requirement was not fixed since it could vary from person to person and from sociocultural context discrepancy ${ }^{[12]}$. Figure 1 was a scatter chart presenting all 591 data points (SD VS. MT) collected during the experiment. It was shown in figure 1 that there was a positive linear trend between two variables when sleep duration is between 6.5 to 7.5 hours. A negative linear trend was found between two variables when sleep duration is between 7.5 to 8.5 hours. Based on the above information, I future hypothesized that Chinese students developed new sleep patterns to reconcile their rests with the highly intense rhythms of learning: they do not need to sleep as long as The National Sleep Foundation recommended to obtain sufficient sleep which could optimize the short-term memory performance. The results of this study can help us better understand Chinese students' sleep and study routines which have habituated to an intense exam-oriented education system. 


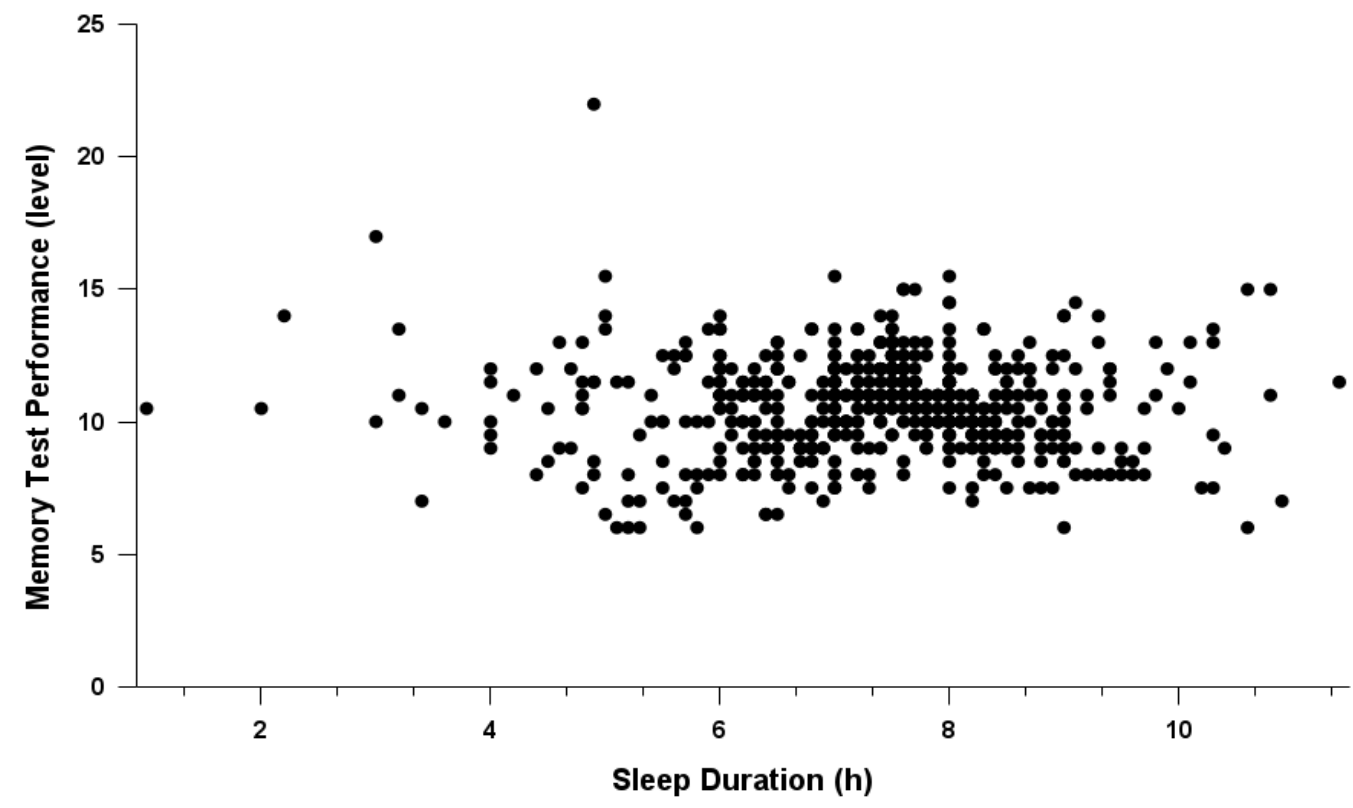

Figure 1. Sleep duration VS. Memory test performance results.

\subsection{Procedure}

Data points in SD VS. MT dataset with sleep duration between 6.5 - 7.5h were divided into group A. Data points in SD VS. MT dataset with sleep duration between 7.5 $8.5 \mathrm{~h}$ were divided into group B. Spearman test was applied to test the correlation between SD and MT in both group A and group B. All data analytical criteria were same to the previous statistical analysis.

\subsection{Results}

Analyses of the spearman test indicated a significantly positive and moderate correlation between SD and MT in group A $(P<0.001$, rho $=0.41)$. Significantly negative and moderate correlations were found between SD and MT in group B $(P<0.001$, rho $=$ $-0.47)$

Sleep duration between 6.5 and 7.5 hours positively correlated with memory test was inconsistent with what was recommended by The National Sleep Foundation. This indicated that participants in this study did not need to sleep that long to have a better short-term memory performance. Therefore, the additional hypothesis was proved.

\section{CONCLUSION}

\subsection{Discussion}

The present study found there is no significant correlation between time to fall asleep and memory test performance. This study also displayed a significantly positive correlation between sleep duration and memory test performance when sleep duration was between 6.5-
$7.5 \mathrm{~h}$, while the significantly negative correlation between sleep duration and memory test performance was found when sleep duration was between 7.5-8.5h. Therefore, it can be concluded that 1)the time to fall asleep did not correlate the short-term memory performance; 2) longer sleep duration positively correlated with short-term memory performance when sleep duration is between 6.5 to7.5 hours; 3) longer sleep duration negatively correlated with short-term memory performance when sleep duration is between 7.5 to 8.5 hours.

\subsection{Implication}

This result has implications for Chinese high school and college students and educators in terms of those students' study-rest schedule and short-term memory performance. Short-term memory is the basis for students to truly grasp the information in lectures ${ }^{[7]}$. The result is effective for students and educators to design a more reasonable sleep schedule that allows students to have optimal short-term memory performance to receive conveyed information in the lectures.

\subsection{Limitations and directions for future research}

A more diverse student background could make the research more conclusive. The study employed convenience samplings, so the high school students who participated in all came from the same international high school in Beijing, China. All of them were planning on applying for universities in countries outside China. Therefore, even though they were living in China having school pressures, the teaching modes, study schedules, class volumes, course contents, and the origin of pressures were still different. Future research should include the 
students from general high schools in China who are planning to participate in the national college entrance examination. Future research is also suggested to include students from different schools and from different provinces in China.

The current study employed self-reporting to collect the data, thus the researcher remained unknown about whether the participants strictly adhered to the rules in which taking the test exactly one hour after they woke up. Therefore, future research can take the volunteers to the lab or choose other methods as long as it allows the researchers to check if the participants adhere to the assigned tasks. Such a monitoring procedure may be possible, as evidenced by the research design of Lo et al. $^{[10]}$.

Unfortunately, the current study did not assess the students' inherent ability. Some participants may be congenitally sensitive to the numbers or had naturally uncanny memories. This made it difficult to evaluate the direct link between sleep patterns and short-term memory performance since they were much more likely to behave well no matter what the sleep pattern was. When the same study is performed, the researchers need to consider this.

This study primarily focuses on the impact of sleep patterns (time to fall asleep and sleep duration) on shortterm memory, which provides implications for future academic research. However, contemporary intelligence theory has identified 76 narrow intellectual abilities while short-term memory is just one of them ${ }^{[14]}$. Therefore, future research needs to evaluate the impacts of those different abilities on school performance and achievement if wanting to provide more accurate instructions to Chinese high school and college students.

As previously mentioned, this study put the data points from high school students and college students in the same data pool and analyzed them together. This design did not allow the study to exclude the differences between high school and college students. Further design is suggested to assign the high school and college students to two different groups and conduct a data analysis exclusively. A larger sample size for each group is recommended.

Finally, future research is needed to support the additional analysis in this study. Additional data analysis in this study is only limited to the SD VS. MT data points with a sleep duration between 6.5-8.5h, however, this may contain an inaccuracy. For participants who had more sleep duration between $6.5-8.5 \mathrm{~h}$ in experimental days, their data points could be included a lot. Yet, participants who always slept for more than $8.5 \mathrm{~h}$ or less than $6.5 \mathrm{~h}$ in those days, their data points could be excluded a lot. This imbalance may compromise the effectiveness of the results.

\section{ACKNOWLEDGMENTS}

The author wishes to thank Dr. Nanthia Suthana for her valuable assistance with the development of the study. The author thanks Yufan Zhou for her critical comments and suggestions. The author thanks all the participants for their cooperation in the data collection.

\section{REFERENCES}

[1] Li, L., Wang, Y. Y., Wang, S. B., Li, L., Lu, L., Ng, C. H., ... \& Xiang, Y. T. (2017). Sleep duration and sleep patterns in Chinese university students: A comprehensive meta-analysis. Journal of Clinical Sleep Medicine, 13(10), 1153-1162.

[2] Sun, W., Yu, Y., Yuan, J., Li, C., Liu, T., Lin, D., ... \& Shan, G. (2015). Sleep duration and quality among different occupations--China national study. PloS one, 10(3), e0117700.

[3] Kang, J. H., \& Chen, S. C. (2009). Effects of an irregular bedtime schedule on sleep quality, daytime sleepiness, and fatigue among university students in Taiwan. BMC public health, 9(1), 1-6.

[4] Ratcliff, R., \& Van Dongen, H. P. (2009). Sleep deprivation affects multiple distinct cognitive processes. Psychonomic bulletin \& review, 16(4), $742-751$

[5] Roth, T. (2007). Insomnia: definition, prevalence, etiology, and consequences. Journal of clinical sleep medicine, 3(5 suppl), S7-S10.

[6] Zhang, J. (2004). Memory process and the function of sleep. Journal of Theoretics, 6(6), 1-7.

[7] Shallice, T. (1982). Specific impairments of planning. Philosophical Transactions of the Royal Society of London. B, Biological Sciences, 298(1089), 199-209.

[8] Diekelmann, S., \& Born, J. (2010). The memory function of sleep. Nature Reviews Neuroscience, 11(2), 114-126.

[9] Stickgold, R., \& Walker, M. P. (2013). Sleepdependent memory triage: evolving generalization through selective processing. Nature neuroscience, 16(2), 139-145.

[10] Lo, J. C., Chong, P. L., Ganesan, S., Leong, R. L., \& Chee, M. W. (2016). Sleep deprivation increases formation of false memory. Journal of sleep research, 25(6), 673-682.

[11] de Winter, J. C., Gosling, S. D., \& Potter, J. (2016). Comparing the Pearson and Spearman correlation coefficients across distributions and sample sizes: A tutorial using simulations and empirical data. Psychological methods, 21(3), 273. 
[12] Hirshkowitz, M., Whiton, K., Albert, S. M., Alessi, C., Bruni, O., DonCarlos, L., ... \& Hillard, P. J. A. (2015). National Sleep Foundation's sleep time duration recommendations: methodology and results summary. Sleep health, 1(1), 40-43.

[13] Steptoe, A., Peacey, V., \& Wardle, J. (2006). Sleep duration and health in young adults. Archives of internal medicine, 166(16), 1689-1692.

[14] Gradisar, M., Terrill, G., Johnston, A., \& Douglas, P. (2008). Adolescent sleep and working memory performance. Sleep and Biological Rhythms, 6(3), 146-154. 